EDUCACIÓN

Y HUMANISMO
Educación y Humanismo 20(34): pp. 173-186. Enero-Junib, DOI: http://dx.doi.org/10.17081/eduhum.20.34.2863

\title{
La evaluación como medio de aprendizaje
}

\section{Evaluation as learning means}

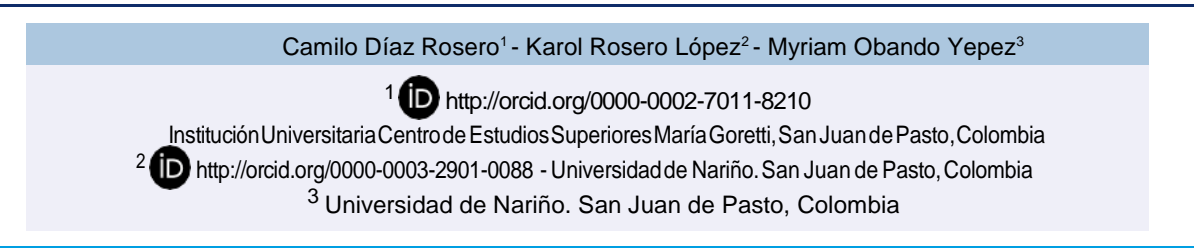

Resumen

Objetivo: El fin de este trabajo es mostrar los efectos pro y en contra de la educación superior colombiana al ser tratada como una mercancía.

Método: Se presenta una revisión de aportes al tema de autores como Rodríguez (2005), Aboites (2007) Ruiz (2009), entre otros. "La Educación Superior en las Negociaciones Internacionales", "Acuerdo General sobre el Comercio de Servicios (GATS) y la Educación Superior", "Aspectos básicos de la Educación Superior en Colombia", "TLC Colombia-Estados Unidos y la Educación Superior", "La experiencia educativa a nivel internacional".

Resultados: Al analizar el sector de la educación en países como Gran Bretaña, Estados Unidos, Australia, Alemania, se observa que es dinámico e importante por ser una actividad que reporta ganancias económicas. En países emergentes como Colombia dominado por una política proteccionista, han abierto sus economías para integrarse a la economía mundial. Para cumplir en el sector de la educación con este proceso de globalización se promulgó la Ley 30 de 1992.

Conclusiones: La educación superior al ser considerada como un servicio, reporta altas ganancias económicas y proliferación de instituciones de dudosa calidad, por lo que el Gobierno debe estar atento y ser más riguroso en su regulación.

Palabras clave: Evaluación, Medios de aprendizaje, Conocimiento.

Abstract

Objective: The purpose of this work is to show the effects pro and against Colombian higher education to be treated as a commodity.

Method: A review of contributions to authors such as Rodríguez (2005), Aboites (2007), Ruiz (2009) and others is presented. "Higher Education in Negotiations Internationals", "General Agreement on Trade in Services (GATS) and Education Superior", "Basic Aspects of Higher Education in Colombia", "TLC Colombia-United States and Higher Education", "The educational experience at the international level".

Results: When analyzing the education sector in countries like Great Britain, United States, Australia, Germany, it is observed that it is dynamic and important for being an activity that reports economic gains. In emerging countries like Colombia dominated by a protectionist policy, they have opened their economies to integrate into the world economy. To comply in the education sector with this process of globalization, Law 30 of 1992 was promulgated.

Conclusions: Higher education, when considered as a service, reports high economic gains and proliferation of institutions of doubtful quality, so that the Government must be attentive and be more rigorous in its regulation.

Key words: Evaluation, Learning means, Knowledge.

Cómo citar este artículo (APA):

Díaz, C., Rosero, K. \& Obando, M. (2018). La evaluación como medio de aprendizaje. Revista Educación y Humanismo, 20(34), 173-186.DOI: http://dx.doi.org/10.17081/eduhum.20.34.2863
UpenAccess:

Editor: Patricia Martínez Barrios Universidad Simón Bolívar

Correspondencia: Camilo Díaz Rosero camilodr10@hotmail.com

Recibido:28-08-16 Aceptado: $12-12-17$ Publicado: 01-01-18

DOI: http://dx.doi org/10.17081 eduhum.20.34.2863 


\section{¿QUÉ ES Y PARA QUE SE EVALÚA?}

La evaluación se puede definir de maneras diversas dependiendo de los objetivos, planes y metas que se pretenda alcanzar, es un proceso que se modifica constantemente y atiende las necesidades generacionales y del contexto.

En la antigüedad, la evaluación era considerada como una comparación de elementos para saber lo que estaba bien o mal, sin embargo actualmente se concibe como el proceso de recolección de evidencias y de formulación de juicios sobre la medida y la naturaleza del progreso hacia los desempeños requeridos, establecidos en un estándar o un resultado del aprendizaje alcanzado (McDonald, 2005, p.230).

Por su parte, Tyler (1950) considera que la evaluación es en sí "el proceso para determinar hasta qué punto los objetivos educativos han sido alcanzados" (p.69), mientras que Lafourcade (1977), la define como "la etapa del proceso educativo que tiene como finalidad comprobar, de manera sistemática y en qué medida se han logrado los objetivos propuestos con antelación” (p.123).

Entendida la educación como un proceso sistemático, esta sin lugar a dudas está destinada a lograr cambios duraderos y positivos en la conducta de los sujetos, integrándolos tomando como base objetivos trazados y definidos en forma concreta, precisa, social e individualmente aceptables.

Benito y Cruz (2007) conciben la evaluación como uno de los elementos claves del proceso formativo en cualquier nivel educativo, cuyo desarrollo y resultados tienen consecuencias en términos formativos, acreditativos e incluso económicos, por lo tanto, es un proceso directamente vinculado con la calidad de la enseñanza: su correcta definición y desarrollo establecen el marco necesario para conducir el aprendizaje, para ajustar los contenidos y métodos de enseñanza y, en último término, para permitir la mejora continua del proceso formativo (p.47).

Por otra parte para Torrado (2000) la evaluación se torna un poco más específica y la asume como un proceso de formación pero por competencias contemplándola como el paso más importante en el proceso de aprendizaje y plantea la relación que tiene la competencia y el logro "El logro no es otra cosa que el conocimiento que se usa, es decir, la competencia. Sobra decir que una competencia puede ser observada en múltiples indicadores" (p.31). 
La competencia tiene sinónimos tales como logro, capacidad, disposición, evidencia, realización, función, demostración y tanto el aprendizaje como el desarrollo de una competencia supone conocimientos, saberes, valores, actitudes y habilidades que emergen de la interacción que se establece entre el individuo y su contexto.

En sintonía con el planteamiento anterior, Tobón (2010) exalta este tipo de evaluación, como aquella que privilegia el desempeño del estudiante en actividades reales o simuladas propias del contexto, y les da mayor relevancia que a las actividades enfocadas en los contenidos académicos (p.119).

Tomando como referencia las anteriores definiciones, la evaluación es entonces un punto de partida donde cobra sentido el proceso educativo, posee una finalidad clara, se ajusta a las realidades del entorno, persigue objetivos y metas, se desarrolla en cualquier momento y lugar, genera espacios reflexivos y al ser un elemento obligado en el quehacer pedagógico se convierte en agente transformador de calidad.

Continuando con nuestro análisis, y dando respuesta a nuestra segunda premisa ¿para qué evaluamos? nos detendremos a analizar algunas funciones específicas de la misma tales como: diagnosticar puesto que permite conocer de primera mano los resultados acerca de la metodología empleada en el proceso de enseñanza y reorientar la práctica pedagógica basada en la toma de decisiones sobre realidades y expectativa de los evaluados.

Retroalimentar: ofreciendo al evaluado la oportunidad de confirmar sus aciertos y clarificar sus errores siendo esta una de las funciones primordiales si se quiere realizar planes de mejoramiento y alcanzar aprendizajes significativos.

Reorientar o redireccionar: haciendo que la atención del evaluado se dirija hacia los aspectos de mayor relevancia donde tuvo mayores dificultades, motivándolo a perfeccionar sus conocimientos.

Concienciar: brindando al evaluado la opción de evidenciar la importancia sobre su grado de avance o nivel de logro en el proceso de aprendizaje.

Reforzar: de manera clara y oportuna las áreas de estudio en el que el aprendizaje haya sido insuficiente a través del diseño de planes de mejoramiento continuo. 
Valorar: de manera cuantitativa y cualitativa asignando calificaciones representativas del aprendizaje obtenido.

Analizar y reajustar: mediante un estudio crítico permite justificar la viabilidad de los programas a la luz de las circunstancias y condiciones reales dando espacio para proponer reajustes y alternativas de cambio fortaleciendo de manera adecuada los aciertos y mejorando las debilidades, permitiendo hacer una revisión sobre la pertinencia de las mismas.

Planear: como consecuencia de los anteriormente expuesto, permite que las nuevas experiencias de aprendizaje partan desde un punto real siendo posible visualizar la secuencia lógica de los temas, como a la coherencia estructural del proceso educativo.

Sin lugar a dudas son muchas más las funciones de la evaluación y son directamente proporcionales a los objetivos que se quieran alcanzar, se hace necesario enfatizar sobre el factor común que encierra esta y su verdadero propósito, el cual es el de incrementar la calidad y en consecuencia, alcanzar el éxito del proceso de enseñanza-aprendizaje a través de una lectura y replanteamiento constante, teniendo en cuenta que los procesos no son estáticos sino que están en constante evolución y cambio, son maleables y se pueden adaptar al contexto real.

Luego entonces, motivará al maestro para realizar una autocrítica, de su quehacer pedagógico, para que tome la evaluación como la principal herramienta en el mejoramiento de los procesos educativos siendo asumida como un todo y no como un mecanismo de represión.

\section{¿CUÁL ES LA FINALIDAD DE LA EVALUACIÓN?}

"La evaluación tiene como propósito determinar en qué medida se están cumpliendo las metas de calidad que se fijan en los estándares, asociadas a los aprendizajes que se espera logren los estudiantes a su paso por la escuela. Por tanto, la evaluación brinda retroalimentación a las instituciones educativas, a las entidades territoriales y al Ministerio de Educación Nacional, detectando fortalezas y debilidades, y valorando el impacto de los procesos educativos sobre el desarrollo de competencias básicas por parte de los estudiantes del país" (Ministerio de Educación Nacional [MEN] (2013). Periódico Altablero. Colombia).

En este sentido, la evaluación es un instrumento diseñado para mejorar de 
una manera continua y permanente el proceso educativo, permite obtener de primera mano información fundamental de los avances presentados a lo largo del desarrollo académico y formativo de los evaluados y con fines pertinentes se aplica en un momento específico, dependiendo lo que se quieraalcanzar.

Al iniciar cualquier proceso educativo, es necesario empezar aplicando un instrumento evaluativo entendido como con el ánimo de recoger de manera puntual, los saberes previos del estudiante, un material, que permita tener un acercamiento real al objeto de conocimiento y a su vez tomar decisiones sobre los contenidos vistos, para que posteriormente puedan ser reajustados de manera precisa y oportuna en el plan de estudios, incorporando algunos temas de interés que colmen expectativas e interrogantes que traen los estudiantes acerca de lo que se está evaluando, este tipo de evaluación se denomina diagnóstica.

Etimológicamente el término diagnóstico (dia-gnosis) se refiere al conocimiento que permite discernir y distinguir (Real Academia Española (2015). Diccionario Etimológico: Madrid). En este caso, el carácter diagnóstico de la evaluación admite analizar y emitir juicios de valor sobre el nivel alcanzado del estudiante y definir cuál será su nuevo punto de partida; Según Brenes (2006), la evaluación diagnóstica "es el conjunto de técnicas y procedimientos evaluativos que se aplican antes y durante el desarrollo del proceso de instrucción" (p.27).

No todos los estudiantes son iguales, todos aprenden de manera diferente, el diagnóstico nos permite analizar a un grupo específico, este tipo de evaluación no debe llevar nota, puesto que no es examen, no necesariamente debe ser escrita, puede ser oral, a través de entrevistas, exposiciones, foros o cualquier otra técnica, ya que busca tener aproximaciones a las realidades educativas, no precisamente tiene que ser individual, puede hacerse en grupo para tener una visión general, obligatoriamente: no tiene que medir conocimientos, puede usarse para evidenciar actitudes individuales y colectivas; por último no tiene que ser solamente para quien evalúa, ya que puede ser abierta y con el propósito de ser retroalimentada, los resultados obtenidos serán un motivo para reforzar los contenidos aprendidos y abrirá un espacio que dará cabida a lo nuevo.

Por otra parte, la actitud que tenga el maestro frente a la evaluación, como instrumento y proceso, el sentido y significado que se le otorgue y la forma como se desligue esta del conocimiento absoluto, serán puntos claves para 
dejar de tomarla como parte independiente de los procesos educativos y se convierta en elemento transversal en todos los ámbitos que involucren situaciones de formación (Álvarez, 2007).

Otro tipo de evaluación es la formativa, la cual tiene como finalidad orientar y mejorar permanentemente el proceso de enseñanza-aprendizaje obteniendo información valiosa sobre el avance académico y actitudinal del evaluado, es procesual y continua, permite detectar cuáles son los aciertos y debilidades para saber con certeza, dónde reajustar los contenidos, es más de fondo ya que permite reestructurar en cualquier momento no solo los contenidos sino también las estrategias de enseñanza, le facilita al evaluador identificar cuáles métodos o estrategias están fallando ya que suministra información sobre los progresos, comprensión y aprendizaje en cualquier etapa o momento.

A diferencia de la evaluación diagnóstica, la formativa tiene la asignación de una nota o calificación que es proporcional a lo demostrado en cada etapa por el evaluado, mediante la aplicación de diferentes estrategias como tareas, revisiones individuales, exposiciones, trabajos escritos, aplicación de exámenes cortos sobre temas puntuales, participación en trabajos de campo y dependiendo del propósito que se desee alcanzar, se pueden diseñar individuales o en grupo.

Rotger (1990) opina que la evaluación formativa requiere de un flujo continuo de información en relación con cada alumno, de esa manera es posible tener una conciencia sobre las fallas del proceso de enseñanza-aprendizaje.

El conocimiento de esta situación por parte del docente será posible debido a la estructuración de un diagnóstico básico de la situación, basado en lo que el autor llama: tres tipos de contenidos básicos: la integración social en el grupo (relación consigo mismo y con sus compañeros), el desarrollo de las actitudes y los conocimientos o destrezas específicas para cada área (p.76).

La evaluación formativa es una herramienta esencial para el evaluador, puesto que cumple doble función, la primera autoevaluar su quehacer pedagógico, permitiéndole evidenciar de manera precisa si tanto la metodología como la didáctica empleadas son las adecuadas y la segunda percibir el grado de receptividad y aceptación de los procesos por parte de los estudiantes y si su nivel de avance es el deseado.

La evaluación sumativa es la que se aplica al final de un período determinado con el fin de comprobar los logros alcanzados en ese lapso de tiempo. 
Permite determinar la valía final del proceso, el grado de aprovechamiento del estudiante y el grado de consecución de los objetivos propuestos. Este tipo de evaluación tiene una función sancionadora en la medida que permite decidir si el estudiante aprueba o no el curso, y si se lo promueve o no al siguiente nivel.

Como consecuencia de este tipo de evaluación, se derivan las escalas de calificaciones, muchas veces puede ser el compendio de muchas actividades que se realizaron a lo largo del proceso, pero por lo general son instrumentos que se diseñan para identificar el acierto y el error.

Sin embargo, también está sujeta a ciertos inconvenientes pues parte del principio que todos los evaluados son iguales, tienen el mismo ritmo de aprendizaje e interactúan de manera similar, entonces se aplican instrumentos estandarizados con ítems fuera de contexto a sabiendas que la realidad escolar es muy diferente.

Este tipo de evaluación, permite la calificación de competencias específicas básicas, como analizar, argumentar y proponer, pero excluye la valoración del ser aunque se hacen escalas para la asignación de una nota numérica a este último aspecto.

Generalmente las estrategias más comunes en los procesos de evaluación sumativa son las siguientes: Asignación de trabajo final, desarrollo de miniproyectos, investigaciones de campo, elaboración de ensayos, aplicación de pruebas finales escritas/orales/mixtas, y la aplicación de pruebas estandarizadas, las más comunes de selección múltiple con una respuesta.

\section{LA EVALUACIÓN COMO MEDIO DE APRENDIZAJE}

La evaluación es uno de los temas con mayor protagonismo en el ámbito educativo, a quien se le ha adjudicado más relevancia e importancia y por supuesto responsabilidad. Entendida como un proceso y no como un suceso la evaluación alcanza su sentido máximo cuando es utilizada como un medio de aprendizaje y no como un fin.

Tradicionalmente siempre se ha evaluado justo al final de cualquier curso académico, dando a entender que este es el camino para encontrar un resultado basado en la prueba del acierto y el error, luego entonces se hace factible afirmar que "a mayor cantidad de aciertos mayor es el grado de asimilación de conceptos" y por consiguiente un estudiante es "bueno" y 
rinde académicamente en la medida que obtenga más respuestas acertadas. Si bien es cierto siempre se ha hecho así y ha funcionado siendo estas las realidades educativas del aula, no necesariamente se está evaluando, simplemente estamos aplicando un instrumento evaluativo llamado examen, prueba, previa, test o quiz, cuyo objetivo es ubicar en una escala previamente concertada con su equivalencia en conceptos o números a un estudiante convirtiéndolo en repetidor de teorías; Construir una prueba en la que los alumnos fracasan es muy fácil, diseñar una prueba para aprender sobre los logros y dificultades de nuestros alumnos es mucho más difícil.

No es lo mismo evaluar para el saber que evaluar para el aprendizaje, si bien es cierto la evaluación como herramienta didáctica es la mejor estrategia para medir conocimientos, mal empleada puede ocasionar apatía en el evaluado si lo hacemos en exceso.

La evaluación debe planificarse y concertarse, tener objetivos claros y definidos, no solo debe buscar la asignación de un puntaje, por el contrario, debe convertirse en un pretexto para propiciar espacios de análisis y síntesis.

Luego entonces abrirá un camino para la retroalimentación, un espacio para la comunicación y la libre expresión; qué importante sería que a la luz del desarrollo de un instrumento evaluativo, se alcancen aprendizajes significativos basados por ejemplo en los desaciertos, pasando de la teoría a la práctica, del concepto a la vivencia, del error al proceso de superación.

Según Amaranti (2010), la retroalimentación es reconocida por los nuevos enfoques de evaluación como una acción crucial para transformar la evaluación en una oportunidad para aprender (p.5).

Bernard (2000) afirma que resulta elocuente observar la forma en que los docentes hacen llegar a sus estudiantes el balance de la evaluación. Para este autor "tal comunicación, en efecto, suele terminar en una calificación tan poco esclarecedora como un dígito o en términos como "insuficiente", "suficiente... sobresaliente"; en definitiva, en una expresión simbólica de la que el estudiante apenas puede extraer información relevante de su conducta como aprendiz" (p.21).

Siguiendo esta misma línea, para Stobart (2005), "la retroalimentación es información que permite al estudiante cerrar la brecha entre el desempeño actual y el deseado, determinar dónde está, dónde quiere llegar y qué es lo que le ayuda a aprender (p.23). 
Si el evaluado se pone en un estado de reconocimiento y autocrítica, reflexionando acerca de sus equivocaciones con el ánimo de superarlos o sus aciertos para fortalecerlos, aprenderá aún más fácil porque partirá desde lo que lo motiva a aprender, argumentará y explicará todo cuanto crea conveniente teniendo en cuenta que no hay nada más atractivo que lo propio y dará pie para darle vida al verdadero significado del rol del maestro como mediador y orientador.

Luego sí, la evaluación toma una importancia específica, se asume como una estrategia útil y necesaria para el mejoramiento continuo haciendo posible el ideal de la calidad educativa.

Ya en la realidad de las instituciones educativas y en los espacios escolares, la evaluación deberá ajustarse y ser coherente con la misión, propósitos, modelo o enfoque pedagógico; si la evaluación se diseña únicamente para repetir conceptos, el aprendizaje se hará de manera memorística, existirán buenos resultados momentáneos y se obtendrán buenas valoraciones pero solo para este espacio de tiempo y esto no garantizará que en un futuro cercano, ese mismo estudiante recuerde algo de lo evaluado.

Entonces, ¿cómo hacer de la evaluación un medio de aprendizaje? Indagando más a profundidad y conscientes de la verdadera importancia que tiene la evaluación en el campo educativo y tratando de contextualizarlo a la realidad de nuestras aulas, añadimos lo que consideramos la respuesta a nuestro tema de estudio y es la aplicabilidad de "la evaluación auténtica”pero, ¿qué es la evaluación auténtica? La definición de este tipo de evaluación es relativamente nueva y se usa de manera general para diferenciar la evaluación de tradición y la nueva evaluación; esta última nace, como alternativa de cambio mucho más atractiva para el evaluado y mucho más real; a sabiendas que por lo general en la gran mayoría de casos una cosa es lo que se enseña y otra la que se evalúa.

Etimológicamente la palabra auténtico proviene del latín authenticus que significa original o que responde a sí mismo; o del griego authentikos que significa primordial o de poder absoluto o aquel que funciona por sí mismo, o se define también como la acreditación de requisitos o circunstancias que en ello concurren y la certificación con que se testifica la identidad y verdad de algo.

Es un instrumento de valoración que se emplea para dar veracidad al bagaje de conocimientos de quien se educa, es una manera de validar la aplicabi- 
lidad de conceptos y de experiencias en un contexto determinado, es evaluar para aprender.

En contraste a lo anterior, Condemarín y Medina (2000) plantean los siguientes principios sobre el tema: La evaluación auténtica constituye una instancia destinada a optimizar la calidad de los aprendizajes, su propósito principal es mejorar y aumentar la probabilidad de que todos los estudiantes aprendan. En este sentido, la evaluación auténtica es una actividad formadora que permite regular los aprendizajes; es decir, comprenderlos, retroalimentarlos y renovar los procesos involucrados en ellos (p.206).

Para conseguir verdaderos aprendizajes, la evaluación debe basarse fundamentalmente en la autoevaluación, la autocrítica y la reflexión constante y es el docente quien debe promover que sean los propios estudiantes los que descubran los criterios de realización de esta tarea; la evaluación auténtica se centra en las competencias que se pretende alcanzar a través del proceso educativo convirtiéndose en consecuencia de la acción de enseñanza.

Por tal motivo debe verse como un medio para alcanzar un propósito, como parte de un todo que nace de manera espontánea y que se evidencia en cualquier espacio, y que tiene lugar cada vez que el estudiante participe, intervenga, socialice o pregunte en las diferentes actividades académicas específicas, donde fluya información continua, donde exista retroalimentación, optimizando los procesos comunicativos y por consiguiente alcanzado la calidad de los procesos.

Además puede considerase como alternativa de cambio en la cultura de la evaluación, donde es claro el diseño de instrumentos de pregunta y respuesta; una evaluación auténtica va más allá que el anterior postulado, ya que busca evaluar lo que se hace, encontrando el punto medio entre práctica y conocimiento.

De igual manera, implica un estado de reflexión por parte del estudiante, pues la meta es la promoción explícita de sus capacidades de autorregulación y reflexión sobre su propio aprendizaje. En este sentido Díaz y Hernández (2002) complementan aseverando que es una evaluación de proceso y formativa, donde son prácticas relevantes la evaluación mutua, la coevaluación y la autoevaluación (p.271).

Aprender de lo evaluado se convierte entonces en una evaluación auténtica, el acto de reconocer fortalezas y debilidades en el desarrollo de lasprácticas 
educativas es ahora una constante, aprender del error es el principio para el inicio de la transformación de pensamientos y paradigmas. Según Astolfi (1997) (citado por Condemarín y Medina, 2000, p.25), los errores son constitutivos del acto mismo de conocer y reflejan un obstáculo epistemológico al que se enfrenta el individuo.

Este obstáculo no constituye un vacío proveniente de la ignorancia; muy por el contrario, surge de los conocimientos previos del individuo, los cuales en un momento dado le impiden construir nuevos conocimientos.

Cuando tomamos como base ese "error" y lo redireccionamos con opciones acertadas, dando apertura a un espacio de diálogo, sin descartar o descalificar ninguna de las intervenciones y por el contrario resaltamos los nuevos aportes que nos servirán posteriormente para ampliar los contenidos y corregirlos puntualmente, obtendremos muchas más producciones intelectuales y alcanzaremos nuevos conceptos dando respuesta no solo a las premisas iniciales, sino que de manera significativa habremos dado pie al análisis a la reflexión y a la síntesis tras la búsqueda de un porqué con argumentos y razones contundentes.

Schön (1998) (citado por Condemarín y Medina, 2000, p.123) señala que los profesores aprenden a enseñar y a mejorar su enseñanza cuando realizan permanentemente "un diálogo inteligente con la práctica", es decir, cuando son capaces de tomar distancia de ella y reflexionar para comprenderla y mejorarla.

La evaluación auténtica incorpora estas apreciaciones porque visualiza el proceso de enseñanza-aprendizaje como mecanismo activo del conocimiento, y al educando como sujeto transformador de cambio que parte desde la reflexión de su propia realidad y demuestra su progreso a través del tiempo con acciones en su desempeño individual o colectivo.

Queda a criterio de cada evaluador el diseño y la aplicabilidad de instrumentos evaluativos que generen transformación; el uso apropiado de técnicas y estrategias didácticas conducirán al buen uso de esta, pero sobre todo cambiarán las concepciones sobre los falsos conceptos de evaluación, como la de sanción, represión, castigo, exclusión, descalificación o mecanismo de opresión, en palabras de Foucault (2002) como un mecanismo para clasificar y castigar (p.140).

\section{CONCLUSIONES}


- Concebida como el principal punto de encuentro para valorar los procesos de aprendizaje, la evaluación es un objeto cambiante capaz de ser adaptado al avance de la sociedad y adecuado a la realidad de los diferentes contextos.

- $\quad$ Cada vez que se aplican pruebas estandarizadas para medir el conocimiento de los educandos cuyos resultados muchas veces no son los esperados, existe en el ambiente una preocupación general por buscar estrategias para el mejoramiento de la calidad educativa, es entonces cuando la evaluación debería jugar un papel importante desde las aulas, los centros e instituciones educativas generando propuestas transformadoras que partan desde la realidad de diferentes contextos, donde se apliquen instrumentos basados en la realidad que permitan el desarrollo de la autonomía y la capacidad para analizar y proponer, que no solo sean contestatarias o den respuestas a preguntas de conocimiento, por el contrario, sean espacios de encuentro de saberes retroalimentados de manera constante, donde se involucren todas las disciplinas, donde no se trabajen las áreas de manera aislada y que los aportes conseguidos sirvan como experiencia significativa y trasciendan del contexto local, al regional y luego al nacional en un proceso cíclico; dicho de otra manera el diseño de una evaluación real para ciudadanos reales, una evaluación holística que genere cambio social y cultural, diseñada, aplicada, analizada y reajustada por los agentes educativos quienes en la marcha son los responsables del mejoramiento y el cambio educativo.

- El aprendizaje es continuo y está en permanente desarrollo, por lo tanto la acción educativa eficaz, debe formularse en las mismas condiciones y en consecuencia la evaluación como parte del proceso, no puede ni debe aplicarse aisladamente. Para alcanzar verdaderos aprendizajes se debe concebir la evaluación como un medio y no como un fin.

- La evaluación debe seguir considerándose como un valioso recurso que nos permita seguir diagnosticando, conociendo, corrigiendo y modificando, aquellos aspectos que son inherentes a la actividad del proceso de enseñanza, dejando en claro que el hecho de evaluar no debe ser considerado como un medio represivo sancionatorio, castigador y mucho menos que se utilice con la intención de descalificar o etiquetar a los estudiantes ante los demás.

- Para una sociedad que cambia permanentemente y tras la búsqueda de opciones que mejoren la calidad educativa, surge como alternativa la evaluación auténtica, la cual se constituye en una herramienta 
primordial cuyo único propósito es el de optimizar el desarrollo de los aprendizajes, aumentando las oportunidades para la adquisición de los nuevos conocimientos a través de la reflexión, la autocrítica y el perfeccionamiento en la capacidad de análisis y síntesis, tomando como punto de referencia la realidad existente, es entonces fundamental el papel del maestro quien deberá propiciar la creación de espacios evaluativos que incluyan diferentes estrategias donde se desarrollen los mecanismos de intervención y comunicación para que el educando sea partícipe y responda a una propuesta para la transformación de su entorno.

\section{REFERENCIAS}

Aboites, H. (2007). Tratado de Libre Comercio y educación superior. El caso de México un antecedente para América Latina. Perfiles Educativos, 21(118), 25-53. Recuperado de http://scielo.unam.mx/pfd/peredu/ v29n118/v29n118a3pdf

Álvarez Méndez, J. (2007). Evaluar para conocer, examinar para excluir. Madrid: Morata.

Amaranti, M. (2010). Evaluación de la educación.Santiago de Chile: Pontificia Universidad Católica de Valparaíso. Facultad de Filosofía y Educación. Escuela de Pedagogía.

Benito, Á. \& Cruz, A. (2007). Nuevas claves para la docencia universitaria. Madrid: Editorial Marcea.

Bernard, J. (2000). Modelo Cognitivo de Evaluación Educativa. Escala de Estrategias de Aprendizaje Contextualizado (ESEAC). Madrid: NARCEA. Disponible en: http://postgrado.una.edu.ve/evaluacionaprendizajes/ paginas/bernadcap2.pdf

Brenes, F. (2006). Evaluación diagnóstica,formativa y sumativa de los aprendizajes. Costa Rica: EUNED.

Carreño, F. (2001). Enfoques y principios teóricos de la evaluación. México: Trillas.

Castillo, S. (2002). Compromisos de la evaluación educativa. Madrid: Prentice-Hall.

Condemarín, M. \& Medina, A. (2000). Evaluación auténtica de los aprendizajes: un medio para mejorar las competencias en lenguaje y comunicación. Santiago de Chile: Andrés Bello.

Díaz B., F. \& Hernández, H. (2000). Estrategias de enseñanza para la promoción de aprendizajes significativos. En Estrategias docentes para un aprendizaje significativo. Una interpretación constructivista. México: McGraw-Hill.

Diccionario de la Lengua Española (2015). Definición etimología. Madrid: Edición del Tricentenario.

Ecourban. (2010). Evaluación ¿Qué es? Disponible en: Instituto Nacional para la Evaluación de la Educación. Cambiar a: Instituto Nacional para 
la Evaluación de la Educación (2010). Evaluación ¿Qué es? Disponible en: http://www.ince.edu.mx/index.php/513-reforma-educativa.

Figueroa, C. (2004). Sistemas de evaluación académica. $1^{\text {a }}$ edición. El Salvador: Universitaria.

Foucault, M. (2002). Vigilar y castigar. Buenos Aires, Argentina: Siglo XXI.

Lafourcade, P. (1977). La evaluación de los aprendizajes. Madrid, España: Cincel.

McDonald, R. (2005). Nuevas perspectivas sobre la evaluación. París, Francia: UNESCO.

Michael, G. (2004). Teachers College Press. Teachers College, Amsterdam: Columbia University.

Ministerio de Educación Nacional [MEN] (2013). Periódico Altablero. Colombia: Magisterio.

Rotger, B. (1990). Evaluación formativa. Madrid, España: Cincel.

Rodríguez, C. (2005). La educación superior frente a los acuerdos de libre comercio: Retos y Oportunidades. Oasis, (10), 321-331. Recuperado de http://www.redalyc.org/pdf/531/53101019.pdf

Ruíz, A. (2009). Impactos de la globalización y del TLC del Perú con Estados Unidos en la educación. Políticas de privitación, espacio público y educación en América Latina. Buenos Aires, Argentina: Concejo Latinoamericano de Ciencias Sociales (CLACSO). Recuperado de http:// bibliotecavirtual.clacso.org/ar/ar/libros/grupos/gentili/cap13pdf

Stobart, G. (2005). Lost in Translation: Moving from principles to policy to practice in formative assessment. Assessment in Education: Principles, Policy \& Practice, 12.

Tobón, S. (2010). Secuencias didácticas, aprendizajes y evaluación de competencias.México: Pearson.

Torrado, M. (2000). Educar para el desarrollo de las competencias. Una propuesta para reflexionar. En D. Bogoya, y otros, Competencias y proyecto pedagógico. Bogotá, D.C.: Universidad Nacional de Colombia.

Tyler, R. (1950). Basic principle of curriculum and instruction. Chicago: Chicago University. 